\title{
Muon capture on the deuteron
}

\section{A. E. Elmeshneb*; Jacek Golak}

M.Smoluchowski Institute of Physics, Jagiellonian University, 30-348, Kraków, Poland

E-mail: Alaashaban2000@yahoo.com

The study of few-nucleon system is interesting and important. It gives a microscopic description of complex systems within the framework of modern concepts of nucleon- nucleon and manybody interactions. Using the muon capture process is an ideal tool to study few-nucleon systems. In this work we plan to investigate the $\mu+d \rightarrow v+n+n$ capture reaction. This reaction is interesting for several reasons. First of all, it offers a testing ground for the nuclear wave functions, which for any nucleon-nucleon (NN) forces can be constructed for such light systems with great accuracy. This reaction, is treated as the decay of the corresponding muonic atom, with the muon initially on the lowest $\mathrm{K}$ shell. The muon binding energy in this atom can be safely neglected and in the initial state we deal essentially with the deuteron and muon at rest. The general formalism for dealing with electroweak reactions on the deuteron will be presented.

The 8th International Workshop on Chiral Dynamics, CD2015 ***

29 June 2015 - 03 July 2015

Pisa,Italy

\footnotetext{
* Speaker.

${ }^{\dagger}$ The author would like to thanks Pisa group for the help and guide on the research.
} 


\section{Introduction}

We present our results for the muon capture reaction: $\mu^{-}+d \rightarrow v_{\mu}+n+n$. Muon capture on light nuclei is one of the best known examples of the weak interaction. Studying these kind of processes enables us to understand the weak nuclear current operator, since ground and scattering states of few-nucleon systems are under control. Typically we study the decay of the muonic atom and deal with hyperfine structure of the atomic levels. We will concentrate on the nuclear matrix elements of the corresponding weak current operator calculated in the momentum space within partial wave decomposition scheme.

In the current operator apart from the relatively well known single nucleon contributions, the twonucleon parts (generated by various meson exchange) play an important role. Their details are not well known and several models should be considered [1]. We will employ various models of NN and $3 \mathrm{~N}$ forces, such as the Bonn B [2] or chiral NNLO potentials [3].

Additionally, we will be able to use for the first time the so-called "improved" chiral nucleonnucleon potentials from the Bochum-Bonn group [4] for the $\mu+d \rightarrow v+n+n$ capture reaction, we will show our predictions for all the available five orders of the chiral expansion and for all the five regulators at each order. The estimate of the total decay rates and differential capture rates as a function of the relative neutron-neutron momentum will be presented. Our results with the single nucleon currents look already very promising and we hope for the improvement in the description of the experimental data, when dominant two-nucleon current operators are included in our framework.

\section{The single nucleon current operator}

The transition amplitude driven by the Fermi form of the interaction Lagrangian (see for example Ref. [5]) and leads to a contraction of the leptonic $\left(\mathscr{L}_{\lambda}\right)$ and nuclear $\left(\mathscr{N}^{\lambda}\right)$ parts in the $S$-matrix element, $S_{f i}[6]$ :

$$
S_{f i}=i(2 \pi)^{4} \delta^{4}\left(P^{\prime}-P\right) \frac{G}{\sqrt{2}} \mathscr{L}_{\lambda} \mathscr{N}^{\lambda}
$$

where $G=1.14939 \times 10^{-5} \mathrm{GeV}^{-2}$ is the Fermi constant (taken from Ref. [1]), and $P\left(P^{\prime}\right)$ is the total initial (final) four-momentum. The well known leptonic matrix element

$$
\mathscr{L}_{\lambda}=\frac{1}{(2 \pi)^{3}} \bar{u}\left(\mathbf{p}_{v}, m_{v}\right) \gamma_{\lambda}\left(1-\gamma_{5}\right) u\left(\mathbf{p}_{\mu}, m_{\mu}\right) \equiv \frac{1}{(2 \pi)^{3}} L_{\lambda}
$$

is given in terms of the Dirac spinors (note that we use the notation and spinor normalization of Bjorken and Drell [7]).

The nuclear part is the essential ingredient of the formalism, and is written as

$$
\mathscr{N}^{\lambda}=\frac{1}{(2 \pi)^{3}}\left\langle\Psi_{f} \mathbf{P}_{f} m_{f}\left|j_{w}^{\lambda}\right| \Psi_{i} \mathbf{P}_{i} m_{i}\right\rangle \equiv \frac{1}{(2 \pi)^{3}} N^{\lambda} .
$$

which $j_{w}^{\lambda}$ is the nuclear weak current operator acting in the matrix element between the initial and final nuclear states as shown in Fig. 1. The matrix element of the nuclear weak current operator 
$j_{w}^{\lambda}$ between the initial and final nuclear states depend on the nucleon incoming (p) and outgoing momentum $\left(\mathbf{p}^{\prime}\right)$ and nucleon spin projections $m$ and $m^{\prime}$ :

$$
\begin{aligned}
& \left\langle\frac{1}{2} m^{\prime}\left|\left\langle\mathbf{p}^{\prime}\left|j_{w}^{\lambda}(1)\right| \mathbf{p}\right\rangle\right| \frac{1}{2} m\right\rangle= \\
& \bar{u}\left(\mathbf{p}^{\prime}, m^{\prime}\right)\left(\left(g_{1}^{V}-2 M g_{2}^{V}\right) \gamma^{\lambda}+g_{2}^{V}\left(p+p^{\prime}\right)^{\lambda}\right. \\
& \left.+g_{1}^{A} \gamma^{\lambda} \gamma^{5}+g_{2}^{A}\left(p-p^{\prime}\right)^{\lambda} \gamma^{5}\right) \tau_{-} u(\mathbf{p}, m),
\end{aligned}
$$

containing nucleon weak form factors, $g_{1}^{V}, g_{2}^{V}, g_{1}^{A}$, and $g_{2}^{A}$, which are functions of the four-momentum transfer squared, $\left(p^{\prime}-p\right)^{2}$. We neglect the small difference between the proton mass $M_{p}$ and neutron mass $M_{n}$ and introduce the average "nucleon mass", $M \equiv \frac{1}{2}\left(M_{p}+M_{n}\right)$. Working with the isospin formalism, we introduce the isospin lowering operator, as $\tau_{-}=\left(\tau_{x}-\mathrm{i} \tau_{y}\right) / 2$.

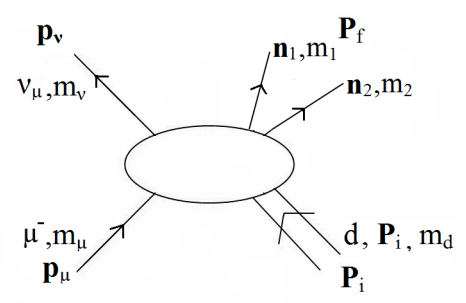

Figure 1: General diagram for muon capture on the deuteron. The details of the lepton-nucleus interaction are hidden inside the ellipse.

\section{Results}

The kinematics of this processes can be treated without any approximations both relativistically and nonrelativistically. Note that here, we use the avarage "nucleon mass" in the kinematics and in solving the Lippmann-Schwinger equation.

In order to evaluate the phase space factor in terms of the relative momentum, The decay rate follows from $S_{f i}$ in a standard way [8] and for the capture process, it is given by

$$
\begin{aligned}
& \Gamma_{d}=\frac{1}{2} G^{2} \frac{1}{(2 \pi)^{2}} \frac{\left(M_{d}^{\prime} \alpha\right)^{3}}{\pi} \int_{0}^{\pi} d \theta_{p_{v}} \sin \theta_{p_{v}} \int_{0}^{2 \pi} d \phi_{p_{v}} \int_{0}^{E_{v}^{\max , n n}} d E_{v} E_{v}^{2} \frac{1}{2} M_{n} p \\
& \int_{0}^{\pi} d \theta_{p} \sin \theta_{p} \int_{0}^{2 \pi} d \phi_{p} \frac{1}{6} \sum_{m_{d}, m_{\mu}} \sum_{m_{1}, m_{2}, m_{v}}\left|L_{\lambda}\left(m_{v}, m_{\mu}\right) N^{\lambda}\left(m_{1}, m_{2}, m_{d}\right)\right|^{2}
\end{aligned}
$$

where the factor $\frac{\left(M_{d}^{\prime} \alpha\right)^{3}}{\pi}$ stems from the $K$-shell atomic wave function, $M_{d}^{\prime}=\frac{M_{d} M_{\mu}}{M_{d}+M_{\mu}}$ and $\alpha \approx \frac{1}{137}$ is the fine structure constant.

Our predictions shown in Fig. 2 calculated with different nucleon-nucleon potentials lie very close to each other. We take the older Bonn B potential [2], the AV18 potential [9] and five different 
parametrizations of the chiral next-to-next-to-leading order (NNLO) potential from the BochumBonn group [10]. The corresponding total $F=\frac{1}{2}$ rates vary only by about $2 \%$, while the total $F=\frac{3}{2}$ rates are even more stable.
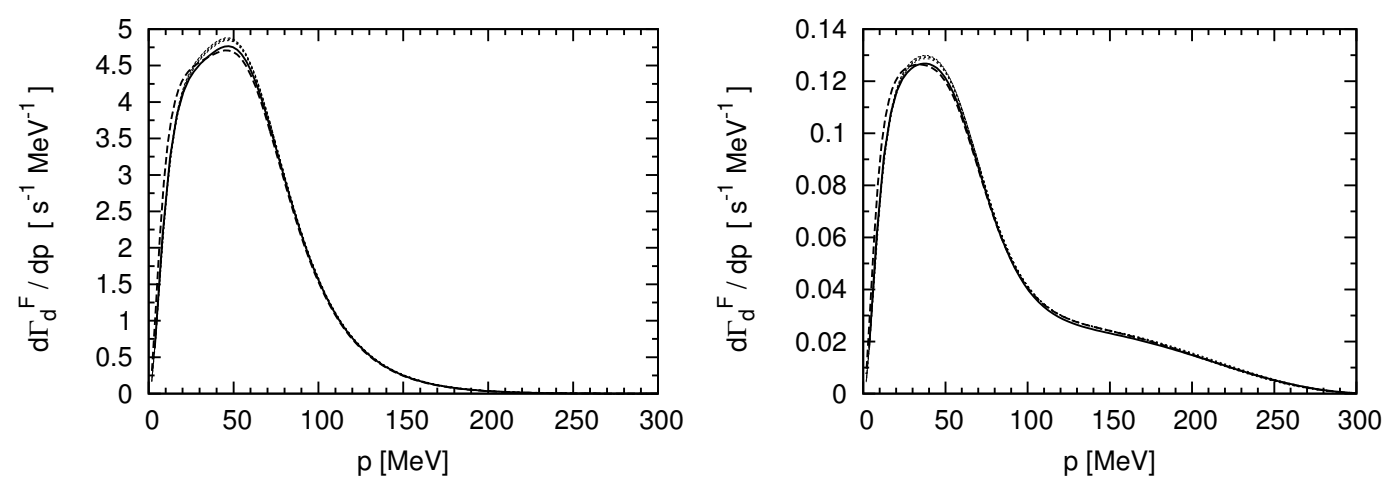

Figure 2: Differential capture rate $d \Gamma_{d}^{F} / d p$ of the $\mu^{-}+{ }^{2} \mathrm{H} \rightarrow v_{\mu}+n+n$ process calculated using standard PWD with various nucleon-nucleon potentials: the AV18 potential [9] (solid curves), the Bonn B potential [2] (dashed curves) and the set of chiral NNLO potentials from Ref. [10] (bands) for $F=\frac{1}{2}$ (left panel) and $F=\frac{3}{2}$ (right panel) as a function of the relative neutron-neutron momentum $p$. Note that the bands are very narrow and thus appear practically as a curve. All the partial wave states with $j \leq 4$ have been included in the calculations with the single nucleon current operator containing the relativistic corrections. Note that the average "nucleon mass" is used in the kinematics and in solving the Lippmann-Schwinger equations.

In Table 1 we show results for the doublet and quadruplet capture rates calculated for all the available five orders of the chiral expansion and for all the five regulators at each order (the socalled "improved"chiral nucleon-nucleon potentials from the Bochum-Bonn group [4]).

\section{Summary}

We show predictions for the differential and total capture rates calculated with quite many different nucleon-nucleon potentials and the single nucleon current operator. The calculation also have done for the quadruplet differential and doublet capture rate. Finally we show for the first time results based on the new, improved chiral potentials from the Bochum-Bonn group [4]. These new nucleon-nucleon forces are available for five orders of the chiral expansion and (at each order) with five different regularizations. Our results demonstrate a very welcome property of the new chiral forces since the range of predictions obtained at a given order but for five different regulators becomes very narrow when going to higher and higher orders of the chiral expansion. The spread of the results at $\mathrm{N} 4 \mathrm{LO}$ is below $0.5 \%$ !

\section{References}

[1] L. Marcucci et al., Phys. Rev. C83, 014002 (2011).

[2] R. Machleidt, Adv. Nucl. Phys. 19 (1989) 189.

[3] E. Epelbaum, Prog. Part. Nucl. Phys. 57, 654 (2006).

[4] E. Epelbaum, H. Krebs, U.-G. Meißner, arXiv:1412.0142 [nucl-th] (2014). 
[5] J.D. Walecka, Theoretical Nuclear and Subnuclear Physics, Oxford University Press, New York, 1995.

[6] R. Skibiński, J. Golak, H. Witała, and W. Glöckle, Phys. Rev. C59, 2384 (1999).

[7] J.D. Bjorken, S.D. Drell, Relativistic Quantum Mechanics, McGraw-Hill Science/Engineering/Math, 1998.

[8] A.D.Matrin, T.D.Spearman, Elementary Particle Theory, North-Holland Publishing Company, 1970.

[9] R.B. Wiringa, V.G.J. Stoks, and R. Schiavilla, Phys. Rev. C 51, 38 (1995).

[10] E. Epelbaum, W. Glöckle, and U.-G. Meißner, Nucl. Phys. A 747, 362 (2005). 
Table 1: Doublet $(F=1 / 2)$ and quadruplet $(F=3 / 2)$ capture rates for the $\mu^{-}+{ }^{2} \mathrm{H} \rightarrow v_{\mu}+n+n$ reaction calculated with the improved chiral nucleon-nucleon potential [4] for different orders of the chiral expansion and the single nucleon current operator with the relativistic corrections (RC). Plane wave results (PW) and results obtained with the rescattering term in the nuclear matrix element (full) are shown. The neutron mass is used in the kinematics and in solving the Lippmann-Schwinger equations. All the partial waves with the total $2 \mathrm{~N}$ angular momentum $j \leq 6$ are employed. In the last column the spread of the full results for $F=1 / 2$ at the given order, $\Delta \Gamma_{d}^{1 / 2} \equiv \Gamma_{d}^{1 / 2} \max -\Gamma_{d}^{1 / 2}$ min , is also presented.

\begin{tabular}{|c|c|c|c|c|c|}
\hline \multirow[b]{3}{*}{ nucleon-nucleon force } & \multicolumn{4}{|c|}{ Capture rate $\Gamma_{d}^{F}$ in $\mathrm{s}^{-1}$} & \multirow[b]{3}{*}{$\Delta \Gamma_{d}^{1 / 2}$ in $\mathrm{s}^{-}$} \\
\hline & \multicolumn{2}{|c|}{$F=1 / 2$} & \multicolumn{2}{|c|}{$F=3 / 2$} & \\
\hline & PW & full & PW & full & \\
\hline LO with $R=0.8 \mathrm{fm}$ & 355.1 & 396.0 & 9.32 & 11.26 & \multirow{5}{*}{3.3} \\
\hline LO with $R=0.9 \mathrm{fm}$ & 357.1 & 397.4 & 9.13 & 11.04 & \\
\hline LO with $R=1.0 \mathrm{fm}$ & 359.1 & 398.4 & 8.94 & 10.89 & \\
\hline LO with $R=1.1 \mathrm{fm}$ & 361.1 & 398.9 & 8.78 & 10.59 & \\
\hline LO with $R=1.2 \mathrm{fm}$ & 362.9 & 399.2 & 8.63 & 10.38 & \\
\hline NLO with $R=0.8 \mathrm{fm}$ & 352.9 & 384.2 & 9.89 & 11.53 & \multirow{5}{*}{5.7} \\
\hline NLO with $R=0.9 \mathrm{fm}$ & 353.8 & 385.8 & 9.88 & 11.53 & \\
\hline NLO with $R=1.0 \mathrm{fm}$ & 354.6 & 387.2 & 9.85 & 11.51 & \\
\hline NLO with $R=1.1 \mathrm{fm}$ & 355.5 & 388.6 & 9.82 & 11.48 & \\
\hline NLO with $R=1.2 \mathrm{fm}$ & 356.3 & 389.8 & 9.77 & 11.45 & \\
\hline N2LO with $R=0.8 \mathrm{fm}$ & 354.2 & 385.0 & 9.83 & 11.60 & \multirow{5}{*}{4.3} \\
\hline N2LO with $R=0.9 \mathrm{fm}$ & 354.9 & 386.1 & 9.84 & 11.56 & \\
\hline $\mathrm{N} 2 \mathrm{LO}$ with $R=1.0 \mathrm{fm}$ & 355.5 & 387.2 & 9.84 & 11.53 & \\
\hline $\mathrm{N} 2 \mathrm{LO}$ with $R=1.1 \mathrm{fm}$ & 356.0 & 388.3 & 9.83 & 11.52 & \\
\hline $\mathrm{N} 2 \mathrm{LO}$ with $R=1.2 \mathrm{fm}$ & 356.6 & 389.3 & 9.82 & 11.50 & \\
\hline N3LO with $R=0.8 \mathrm{fm}$ & 353.0 & 386.8 & 9.70 & 11.44 & \multirow{5}{*}{3.6} \\
\hline N3LO with $R=0.9 \mathrm{fm}$ & 352.8 & 386.4 & 9.74 & 11.48 & \\
\hline $\mathrm{N} 3 \mathrm{LO}$ with $R=1.0 \mathrm{fm}$ & 353.1 & 385.2 & 9.81 & 11.52 & \\
\hline N3LO with $R=1.1 \mathrm{fm}$ & 353.8 & 384.3 & 9.91 & 11.58 & \\
\hline N3LO with $R=1.2 \mathrm{fm}$ & 354.5 & 383.2 & 10.05 & 11.66 & \\
\hline N4LO with $R=0.8 \mathrm{fm}$ & 353.1 & 385.5 & 9.77 & 11.51 & \multirow{5}{*}{1.7} \\
\hline N4LO with $R=0.9 \mathrm{fm}$ & 354.0 & 386.1 & 9.78 & 11.50 & \\
\hline N4LO with $R=1.0 \mathrm{fm}$ & 354.8 & 386.3 & 9.81 & 11.50 & \\
\hline N4LO with $R=1.1 \mathrm{fm}$ & 355.4 & 385.6 & 9.88 & 11.54 & \\
\hline N4LO with $R=1.2 \mathrm{fm}$ & 355.8 & 384.6 & 10.00 & 11.61 & \\
\hline
\end{tabular}

